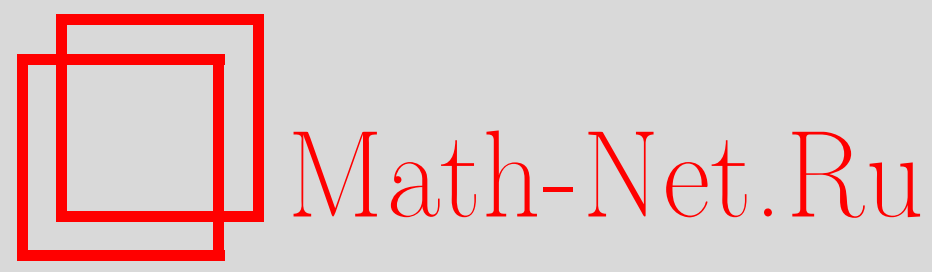

Д. С. Малышев, Экстремальные множества графов при решении задачи демаркации в семействе наследственно замкнутых классов графов, Дискрет. матем., 2012, том 24, выпуск 4, 91-103

DOI: https://doi.org/10.4213/dm1213

Использование Общероссийского математического портала Math-Net.Ru подразумевает, что вы прочитали и согласны с пользовательским соглашением http://www . mathnet.ru/rus/agreement

Параметры загрузки:

IP: 3.89 .185 .249

26 апреля 2023 г., 16:59:41 
УДК 519.17

\title{
Экстремальные множества графов при решении задачи демаркации в семействе наследственно замкнутых классов графов
}

\author{
() 2012 г. Д. С. Малышев
}

\begin{abstract}
В данной работе рассматриваются понятия минимального сложного и граничного классов. Данные классы играют особую роль при определении границы между полиномиальной разрешимостью и $N P$-полнотой задач на графах в семействе наследственных классов. Ранее в одной из работ автора было установлено, что для некоторого типа задач на графах минимальных сложных классов нет. В данной работе, наоборот, доказывается достаточное условие существования таких классов. Его действенность подтверждается новыми примерами минимальных сложных классов и граничных классов для некоторых задач на графах. Для выявленных задач удается полностью описать множества минимальных сложных и граничных классов. Ранее ни для одной задачи на графах таких описаний получить не удавалось.

Работа выполнена при поддержке РФФИ, проекты 10-01-00357-а и 11-01-00107-a, и ФЦП «Научные и научно-педагогические кадры инновационной России на 20092012 гг.», ГК 16.740.11.0310.
\end{abstract}

\section{1. Введение}

На настоящее время накоплено большое количество результатов про полиномиальную разрешимость и $N P$-полноту различных задач на графах во многих классах графов. Мотивы получения новых сведений такого рода могут быть самими разнообразными, но можно выделить два наиболее распространенных:

(1) поиск более широких “простых” классов, объемлющих ранее известные;

(2) поиск $N P$-полных сужений для известных “сложных” случаев.

Вместе с тем, при рассмотрении представительных семейств классов графов можно ставить задачи более общего характера, чем анализ сложности для индивидуального класса. В частности, можно поставить целью выявление пределов, до которых возможны расширения полиномиальной сложности и сужения с “противоположным” сложностным статусом. Тем самым, речь фактически идет о нахождении границы между “простыми” и “сложными” классами из рассматриваемого семейства. 
В настоящем исследовании изучается эта граница для некоторых задач на графах в семействе наследственных классов графов, то есть классов графов, замкнутых относительно удаления вершин. Наследственный класс графов $\mathscr{X}$ определяется множеством своих запрещенных порожденных подграфов $\mathscr{Y}$, что записывается так:

$$
\mathscr{X}=\operatorname{Free}(\{\mathscr{S}\}) .
$$

Дадим строгие определения неформально понимаемым в предыдущих абзацах понятиям простого и сложного классов графов. Пусть $П-$ какая-либо $N P$-полная задача на графах. Наследственный класс графов назовем П-простым, если задача П для графов из этого класса полиномиально разрешима, и П-сложным в противном случае. Далее везде предполагаем справедливость неравенства $P \neq N P$ и не включаем его явно в формулировки полученных результатов.

Естественной идеей решения задачи демаркации является поиск максимальных П-простых и минимальных П-сложных классов, то есть тупиковых классов графов соответствующей сложности из рассматриваемой решетки. К сожалению, использование понятия максимального простого класса графов оказывается безрезультатным. Так, В. Е. Алексеев в [5] установил, что ни один П-простой класс не является максимальным простым (хотя там это утверждается только для задачи о независимом множестве, все рассуждения легко переносятся на общий случай). Вместе с тем, до недавнего времени про минимальные сложные классы ничего не было известно.

Первый результат о подобного рода классах был получен автором в [1], где рассматривалась задача распознавания принадлежности наследственному классу графов и было доказано, что для любой задачи такого типа минимальных сложных классов нет. В той же работе были найдены первые примеры минимальных сложных классов графов. Другие примеры таких классов были найдены в [3, 4] Однако в [1, 3, 4] рассматривалась одна и та же задача о списковом ранжировании, и до результатов настоящей работы не было известно других примеров существования минимальных сложных классов.

Новые такие классы выявляются в данной статье. Вместе с тем, по аналогии с результатами из [1], и для случаев существования минимальных сложных классов хотелось бы получить достаточные условия. Данное условие формулируется в настоящей работе. Оно использует понятие числа Дилворта наследственного класса, введенного в данной статье. Действенность полученного утверждения подтверждается выявленной последовательностью задач на графах $\left\{\Pi_{k}\right\}$, для каждой из которых указывается минимальный $\Pi_{k}$-сложный класс с числом Дилворта, равным $k$. Более того, для любого $k$ данная работа содержит описание всех минимальных $\Pi_{k}$-сложных классов. Для каждого $k$ их оказывается конечное число. Это первые примеры задач на графах с полностью известным множеством минимальных сложных классов.

Итак, для некоторых задач на графах существуют минимальные сложные классы. Таким образом, желание нащупать границу эффективной разрешимости таких задач в семействе наследственных классов неизбежно приводит к рассмотрению пределов убывающих последовательностей из сложных классов. При этом возникает понятие граничного класса графов. Именно, класс графов $\mathscr{X}$ называется П-граничным, если существует такая бесконечная последовательность П-сложных классов графов $\mathscr{X}_{1} \supseteq \mathscr{L}_{2} \supseteq \ldots$, что

$$
\mathscr{X}=\bigcap_{i=1}^{\infty} \mathscr{X}_{i},
$$

и $\mathscr{L}$ является минимальным по включению классом с этим свойством. Понятие граничного класса было введено В. Е. Алексеевым в [5]. Значение этого понятия состоит в том, что 
класс $\mathscr{X}=\operatorname{Free}(\{\mathscr{Y}\})$ с конечным множеством $\mathscr{S}$ является П-сложным тогда и тогда, когда $\mathscr{\mathscr { L }}$ включает некоторый П-граничный класс. Этот результат подтолкнул к попыткам дать полное описание таких классов для ряда графовых задач. В числе соответствующих исследований укажем диссертации $[2,9,11]$ и работы $[5,6,7,10]$. Однако центральная проблема теории граничных классов графов (получение полного описания) до настоящего времени не была решена ни для одной задачи. В этой статье полностью описываются все такие классы для задачи $\Pi_{k}$. Это множество полностью совпадает с соответствующим множеством минимальных $\Pi_{k}$-сложных классов.

В статье приняты следующие обозначения:

$K_{i}$ - полный граф с $i$ вершинами;

$P_{i}-$ простой путь с $i$ вершинами;

$C_{i}-$ простой цикл с $i$ вершинами;

$K_{p, q}$ - полный двудольный граф с $p$ вершинами в одной доле и $q$ вершинами в другой; $K_{i}-e-$ результат удаления из графа $K_{i}$ произвольного ребра;

$G_{1} \oplus G_{2}$ - результат объединения двух графов $G_{1}$ и $G_{2}$ с непересекающимися множествами вершин;

$k G-$ граф, изоморфный графу $\underbrace{G \oplus G \oplus \ldots \oplus G}_{k \text { слагаемых }}$;

$\bar{G}-$ дополнительный граф к графу $G$;

$M^{n}-n$-я декартова степень множества $M$;

$\mathbf{Z}_{+}$- множество неотрицательных целых чисел.

\section{2. Наследственные классы графов с небольшим значением числа Дилворта}

В начале настоящего раздела приведем некоторые определения, связанные с отношениями порядка и их свойствами. Напомним, что любое рефлексивное, антисимметричное и транзитивное отношение $R$, заданное над некоторым универсом $U$, называется отношением порядка. Два элемента $x \in U$ и $y \in U$ называются сравнимыми, если $x R y$ или $y R x$, и несравнимыми в противном случае. Отношение порядка $R$ называется линейным, если любые два элемента из $U$ сравнимы между собой. Подмножество множества $U$ называется цепью, если любые два элемента из этого подмножества являются сравнимыми. Любое подмножество попарно несравнимых элементов из $U$ называется антицепью.

Для конкретной пары $(U, R)$ можно вычислить мощность наибольшей антицепи (то есть максимально возможное количество элементов в антицепях). Обозначим этот параметр через $w(R)$. Возможно, что $w(R)=\infty$ (такая ситуация может иметь место, если $U$ бесконечно). С другой стороны, можно подсчитать наименьшее количество цепей, покрывающих $U$. Обозначим эту характеристику через $c(R)$. Другими словами, $c(R)-$ минимальное количество таких цепей $U_{1}, U_{2}, \ldots$, что каждый элемент из $U$ принадлежит хотя бы одной из этих цепей. Между характеристиками $w(R)$ и $c(R)$ имеются определенные связи. 
Очевидно, что если $c(R)$ и $w(R)$ конечны, то $w(R) \leqslant c(R)$. Также понятно, что если $w(R)$ бесконечно, то и $c(R)=\infty$. Вместе с тем, если $w(R)<\infty$, то числа $w(R)$ и $c(R)$ равны между собой (этот результат доказан в [12]). В этом случае $w(R)$ принято называть числом Дилворта, которое обозначается через $\operatorname{Dil}(R)$. Если $w(R)=\infty$, то число Дилворта $\operatorname{Dil}(R)$ полагается равным бесконечности.

Данный раздел посвящен изучению значений чисел Дилворта применительно к некоторому порядку над графами из наследственных классов. Так, мы всегда будем полагать, что $R$ - отношение “быть порожденным подграфом” над $U=\mathscr{X}$, где $\mathscr{X}-$ некоторый наследственный класс. Для таких $R$ и $U$ соответствующее число Дилворта обозначается через $\operatorname{Dil}(\mathscr{X})$. Этот параметр будем называть числом Дилворта класса $\mathscr{X}$.

Наследственные классы графов с конечным числом Дилворта играют особую роль при систематическом поиске минимальных сложных классов. Поэтому нашей первой целью будет описать все наследственные классы с малым значением величины Dil(æ).

Лемма 1. Число Дилворта класса $\mathscr{X}$ равно единице тогда и только тогда, когда $\mathscr{X}$ состоит только из полных графов или только из пустых графов.

Доказательство. Поскольку

$$
\operatorname{Dil}(\mathscr{X})=1
$$

графы из $\mathscr{X}$ являются линейно упорядоченным множеством. Так как графы $K_{2}$ и $2 K_{1}$ несравнимы, либо $\mathscr{X} \subseteq$ Free $\left(\left\{K_{2}\right\}\right)$, либо $\mathscr{X} \subseteq$ Free $\left(\left\{2 K_{1}\right\}\right)$. В первом случае $\mathscr{X}$ составляют только пустые графы, а во втором - только полные.

Лемма 2. Равенство

$$
\operatorname{Dil}(\mathscr{X})=2
$$

имеет место тогда и только тогда, когда

(1) класс $\mathscr{L}$ состоит из некоторого количества (возможно, бесконечного) клик и некоторого количества (возможно, бесконечного) графов вида $K_{p} \oplus K_{1}$;

(2) справедливо одно из следующих равенств:

$$
\mathscr{X}=\left\{K_{1}, K_{2}, K_{3}, 2 K_{1}, K_{1} \oplus K_{2}, 2 K_{2}\right\},
$$

или

$$
\mathscr{X}=\left\{K_{1}, K_{2}, K_{3}, 2 K_{1}, K_{1} \oplus K_{2}, 2 K_{2}, K_{2} \oplus K_{3}\right\},
$$

или

$$
\mathscr{X}=\left\{K_{1}, K_{2}, K_{3}, 2 K_{1}, K_{1} \oplus K_{2}, 2 K_{2}, K_{2} \oplus K_{3}, 2 K_{3}\right\} ;
$$

(3) класс $\mathscr{X}$ состоит из некоторого количества (возможно, бесконечного) пустых графов и некоторого количества (возможно, бесконечного) графов вида $K_{2} \oplus p K_{1}$;

(4) класс $\mathscr{X}$ состоит из некоторого количества (возможно, бесконечного) пустых графов и графов $K_{2} \oplus K_{1}$ и $2 K_{2}$; 
(5) справедливо одно из следующих равенств:

$$
\mathscr{L}=\left\{K_{1}, 2 K_{1}, 3 K_{1}, K_{2} \oplus K_{1}, 2 K_{2}, 2 K_{2} \oplus K_{1}\right\} \text {, }
$$

или

$$
\mathscr{X}=\left\{K_{1}, 2 K_{1}, 3 K_{1}, K_{2} \oplus K_{1}, 2 K_{2}, 2 K_{2} \oplus K_{1}, 3 K_{2}\right\}
$$

(6) класс $\mathscr{X}$ состоит из некоторого числа (возможно, бесконечного) полных графов и некоторого количества (возможно, бесконечного) пустых графов;

(7) класс $\mathscr{X}$ состоит из не менее чем двух графов вида $K_{1, p}$ и всевозможных их порожденных подграфов;

(8) класс $\mathscr{X}$ состоит из некоторого количества (возможно, бесконечного) пустых графов и графов $K_{2}$ и $P_{3}$;

(9) класс $\mathscr{X}$ состочт из некоторого количества (возможно, бесконечного) пустых графов и графов $K_{2}, P_{3}$ и $K_{1,3}$;

(10) класс $\mathscr{X}$ состоит из некоторого количества (возможно, бесконечного) пустых графов и графов $K_{2}, P_{3}$ и $C_{4}$;

(11) справедливо включение

$$
\mathscr{X} \subseteq\left\{K_{1}, 2 K_{1}, 3 K_{1}, K_{1,3}, K_{2}, P_{3}, C_{4}, K_{2,3}, K_{3,3}\right\} ;
$$

(12) класс $\mathscr{X}$ состоит из некоторого числа (возможно, бесконечного) полных графов и некоторого количества (возможно, бесконечного) графов вида $K_{p}-e$;

(13) класс $\mathscr{X}$ состоит из некоторого числа (возможно, бесконечного) полных графов и графов $\mathrm{P}_{3}$ и $\mathrm{C}_{4}$;

(14) справедливо равенство

$$
\mathscr{X}=\left\{K_{1}, 2 K_{1}, P_{3}, C_{4}, K_{2}, K_{3}, K_{4}-e\right\} .
$$

Доказательство. Из предыдущей леммы следует, что $\mathscr{X}$ обязательно содержит графы $K_{2}$ и $2 K_{1}$. Возможны два случая.

Пусть $K_{1} \oplus K_{2} \in \mathscr{X}$. Тогда графы $K_{3}$ и $3 K_{1}$ не могут одновременно принадлежать $\mathscr{X}$, так как $\left\{K_{3}, 3 K_{1}, K_{1} \oplus K_{2}\right\}$ есть антицепь. Поэтому либо $\mathscr{X} \subseteq \operatorname{Free}\left(\left\{K_{3}\right\}\right)$, либо $\mathscr{L} \subseteq$ Free $\left(\left\{3 K_{1}\right\}\right)$. Рассмотрим возможные случаи.

Если $K_{3} \in \mathscr{X}$, то $3 K_{1} \notin \mathscr{X}$ и $P_{3} \notin \mathscr{X}$ (так как $\left\{K_{3}, 3 K_{1}, P_{2} \oplus K_{1}\right\}$ и $\left\{K_{3}, P_{3}, K_{1} \oplus K_{2}\right\}-$ антицепи). Но тогда $\mathscr{X} \subseteq \operatorname{Free}\left(\left\{3 K_{1}, P_{3}\right\}\right)$. Ясно, что каждый граф из $\mathscr{X}$ имеет не более чем две компоненты связности и каждая из них является кликой. Графы $K_{4}, 2 K_{2}, K_{1} \oplus K_{3}$ образуют антицепь, поэтому они одновременно $\mathscr{X}$ принадлежать не могут. Если $2 K_{2} \notin \mathscr{X}$, то $\mathscr{X}$ содержит только клики и графы вида $K_{p} \oplus K_{1}$ (реализуется п. 1 ). Каждый такой класс имеет число Дилворта, равное 2 . Если $K_{1} \oplus K_{3} \notin \mathscr{X}$, то $X$ содержит только клики и графы $2 K_{1}, K_{1} \oplus K_{2}$ (снова реализуется п. 1 ). Классы такого типа также имеют число Дилворта, равное 2 . Наконец, если $K_{4} \notin \mathscr{X}$, то либо

$$
\mathscr{X}=\left\{K_{1}, K_{2}, K_{3}, 2 K_{1}, K_{1} \oplus K_{2}\right\}
$$


или

$$
\mathscr{X}=\left\{K_{1}, K_{2}, K_{3}, 2 K_{1}, K_{1} \oplus K_{2}, K_{1} \oplus K_{3}\right\}
$$

(опять реализуется п. 1), либо

$$
\mathscr{X}=\left\{K_{1}, K_{2}, K_{3}, 2 K_{1}, K_{1} \oplus K_{2}, 2 K_{2}\right\}
$$

или

$$
\mathscr{L}=\left\{K_{1}, K_{2}, K_{3}, 2 K_{1}, K_{1} \oplus K_{2}, 2 K_{2}, K_{2} \oplus K_{3}\right\}
$$

или

$$
\mathscr{X}=\left\{K_{1}, K_{2}, K_{3}, 2 K_{1}, K_{1} \oplus K_{2}, 2 K_{2}, K_{2} \oplus K_{3}, 2 K_{3}\right\}
$$

(реализуется п. 2).

Если $3 K_{1} \in \mathscr{X}$, то $K_{3} \notin \mathscr{X}$ и $P_{3} \notin \mathscr{X}$ (так как $\left\{3 K_{1}, K_{3}, K_{1} \oplus K_{2}\right\}$ и $\left\{3 K_{1}, P_{3}, K_{1} \oplus K_{2}\right\}$ - антицепи). Тогда $\mathscr{X} \subseteq \operatorname{Free}\left(\left\{K_{3}, P_{3}\right\}\right)$. Отсюда следует, что каждый граф из $\mathscr{X}$ изоморфен графу $p K_{2} \oplus q K_{1}$. Если $4 K_{1} \in \mathscr{X}$, то либо $2 K_{2} \notin \mathscr{X}$, либо $K_{2} \oplus 2 K_{1} \notin \mathscr{X}$. В первом случае $\mathscr{X}$ состоит только из пустых графов и графов вида $K_{2} \oplus p K_{1}$ (реализуется п. 3). Число Дилворта каждого такого класса действительно равно 2. Во втором случае либо реализуется п. 3 , либо $\mathscr{X}$ содержит только пустые графы и только графы $K_{2} \oplus K_{1}, 2 K_{2}$ (реализуется п. 4). Число Дилворта каждого такого класса также равно 2. Если же $\mathscr{Q} \subseteq$ Free( $\left.\left\{4 K_{1}\right\}\right)$, то либо

$$
\mathscr{X}=\left\{K_{1}, 2 K_{1}, 3 K_{1}, K_{2} \oplus K_{1}\right\}
$$

или

$$
\mathscr{X}=\left\{K_{1}, 2 K_{1}, 3 K_{1}, K_{2} \oplus K_{1}, 2 K_{2}\right\}
$$

(реализуется п. 3), либо

$$
\mathscr{X}=\left\{K_{1}, 2 K_{1}, 3 K_{1}, K_{2} \oplus K_{1}, 2 K_{2}, 2 K_{2} \oplus K_{1}\right\}
$$

или

$$
\mathscr{X}=\left\{K_{1}, 2 K_{1}, 3 K_{1}, K_{2} \oplus K_{1}, 2 K_{2}, 2 K_{2} \oplus K_{1}, 3 K_{2}\right\}
$$

(реализуется п. 5). Для всех данных случаев $\operatorname{Dil}(\mathscr{X})=2$.

Будем теперь считать, что $K_{1} \oplus K_{2} \notin \mathscr{X}$. Тогда все несвязные графы из $\mathscr{X}$ являются пустыми. Графы $3 K_{1}, P_{3}$ и $K_{3}$ не могут одновременно принадлежать классу $\mathscr{X}$. Если $\mathscr{X} \subseteq \operatorname{Free}\left(\left\{P_{3}\right\}\right)$, то $\mathscr{X}$ состоит только из полных и пустых графов (реализуется п. 6). Все такие классы графов имеют число Дилворта, равное 2. Пусть теперь $P_{3} \in \mathscr{X}$. Рассмотрим возможные случаи.

Если $3 K_{1} \in \mathscr{X}$, то $\mathscr{X} \subseteq \operatorname{Free}\left(\left\{K_{3}, K_{1} \oplus K_{2}\right\}\right)$. Легко проверить, что все связные графы из $\mathscr{X}$ являются полными двудольными. Если $4 K_{1} \in \mathscr{X}$, то либо $K_{1,3} \notin \mathscr{X}$, либо $C_{4} \notin \mathscr{X}$. Класс 
$\operatorname{Free}\left(\left\{K_{3}, K_{1} \oplus K_{2}, C_{4}\right\}\right)$ состоит из лесов. Поэтому, если $C_{4} \notin \mathscr{X}$, то либо реализуется п. 7, либо $\mathscr{L}$ содержит только пустые графы и только графы $K_{2}, P_{3}$ (реализуется п. 8), либо $\mathscr{X}$ содержит только пустые графы и только графы $K_{2}, P_{3}$ и $K_{1,3}$ (реализуется п. 9). Все классы такого вида имеют число Дилворта, равное 2. Если $C_{4} \in \mathscr{X}$, то $K_{1,3} \notin \mathscr{X}$, поэтому $\mathscr{X} \subseteq \operatorname{Free}\left(\left\{K_{1,3}, K_{3}, K_{1} \oplus K_{2}\right\}\right)$. Но тогда $\mathscr{L}$ содержит только пустые графы и только графы $K_{2}, P_{3}$ и $C_{4}$ (реализуется п. 10). Такие классы имеют число Дилворта, равное 2. Если $4 K_{1} \notin \mathscr{X}$, то $\mathscr{X} \subseteq\left\{K_{1}, 2 K_{1}, 3 K_{1}, K_{1,3}, K_{2}, P_{3}, C_{4}, K_{2,3}, K_{3,3}\right\}$ (реализуется п. 11). Легко проверить, что все такие $\mathscr{L}$ имеют число Дилворта, равное 2.

Если $K_{3} \in \mathscr{X}$, то $\mathscr{X} \subseteq \operatorname{Free}\left(\left\{3 K_{1}\right\}\right)$. Поскольку все графы из $\mathscr{X} \cap \operatorname{Free}\left(\left\{K_{3}\right\}\right)$ являются полными двудольными, то связными графами из $\mathscr{X} \cap \operatorname{Free}\left(\left\{K_{3}\right\}\right)$ могут быть только графы $K_{2}, P_{3}, C_{4}$. С другой стороны, все графы из $\mathscr{X}$, содержащие $K_{3}$, являются кликами или имеют вид $K_{p}-e$. В этом несложно убедиться, учитывая включение $\mathscr{X} \subseteq \operatorname{Free}\left(\left\{K_{1} \oplus K_{2}, 3 K_{1}\right\}\right)$. Графы $K_{4}, C_{4}, K_{4}-e$ одновременно не могут принадлежать классу $\mathscr{X}$ (так как $\left\{K_{4}, C_{4}, K_{4}-e\right\}-$ антицепь). Если $C_{4} \notin \mathscr{X}$, то $\mathscr{X}$ содержит только полные графы и только графы вида $K_{p}-e$ (реализуется п. 12). Все данные классы графов действительно имеют число Дилворта, равное 2 . Если $C_{4} \in \mathscr{X}$ и $K_{4}-e \notin \mathscr{X}$, то $\mathscr{X}$ содержит только полные графы и только графы $P_{3}$ и $C_{4}$ (реализуется п. 13). Число Дилворта этих классов равно 2. Если $C_{4} \in \mathscr{X}$ и $K_{4} \notin \mathscr{X}$, то либо

$$
\mathscr{X}=\left\{K_{1}, 2 K_{1}, P_{3}, C_{4}, K_{2}, K_{3}\right\}
$$

(опять реализуется п. 13), либо

$$
\mathscr{X}=\left\{K_{1}, 2 K_{1}, P_{3}, C_{4}, K_{2}, K_{3}, K_{4}-e\right\}
$$

(реализуется п. 14).

Таким образом, для случаев 1-14, и только для них, справедливо равенство

$$
\operatorname{Dil}(\mathscr{X})=2 \text {. }
$$

Скрупулезный анализ, проведенный при доказательстве леммы 2, показывает, что получение описания классов $\mathscr{L}$ с малым числом Дилворта в терминах имеющихся в них графов, по-видимому, неосуществимо уже при $\operatorname{Dil}(\mathscr{X}) \geqslant 3$. Вместе с тем, наблюдение о том, что классы графов с числом Дилворта $k+1$ могут быть тесно связаны с классами, имеющими число Дилворта $k$, приводит к мысли дать соответствующее индуктивное описание. База такого описания уже заложена в формулировке леммы 1.

Очевидно, что класс графов $\mathscr{X}$ является непосредственным предшественником класса У (по отношению включения), если существует такой граф $G \in \operatorname{Forb}(\mathscr{L})$, что У получается добавлением $G$ к классу $\mathscr{X}$. Ясно, что

$$
\operatorname{Dil}(\mathscr{X}) \leqslant \operatorname{Dil}(\mathscr{y}) \leqslant \operatorname{Dil}(\mathscr{X})+1 .
$$

Вместе с тем, предпринимавшиеся автором попытки охарактеризовать случаи перехода от $\mathscr{X}$ к $\mathscr{y}$ с сохранением числа Дилворта в терминах только окрестности графа $G$ (то есть порожденных подграфов $G$ ) ни к чему не привели. Возможно, такой подход вообще обречен на неудачу. Выходом из этого тупика явился глобальный анализ ситуации, то есть формулировка критерия, в котором фигурирует все семейство $\mathscr{X}$, а не отдельная его часть. Следующее утверждение может быть легко доказано.

Лемма 3. Равенство

$$
\operatorname{Dil}(\mathscr{y})=\operatorname{Dil}(\mathscr{X})
$$


имеет место тогда и только тогда, когда для класса $\mathscr{X}$ существует такое покрытие $\operatorname{Dil}(\mathscr{X})$ цеепями, в котором одна из ичепей целиком состоит из порожденных подграфов графа $G$.

Весьма интересны вопросы о существовании классов графов $\mathscr{X}$, для которых добавление любого графа из Forb $\mathscr{L}$ ) не изменяет (увеличивает) число Дилворта. Ответы на оба вопроса положительны, и ответить на первый вопрос нам помогает лемма 3.

Для любого $k$ в качестве класса $\mathscr{X}$ рассмотрим множество графов с не более чем $k$ вершинами. Понятно, что $\operatorname{Dil}(\mathscr{X})<\infty$ и что $\operatorname{Forb}(\mathscr{X})$ составляют всевозможные графы, имеющие ровно $k+1$ вершину. Понятно, что для любого графа $G \in \operatorname{Forb}(\mathscr{L})$ и любого оптимального покрытия класса $\mathscr{X}$ существует цепь, целиком состоящая из порожденных подграфов графа $G$. Поэтому в силу леммы 3 справедливо равенство

$$
\operatorname{Dil}(\mathscr{X})=\operatorname{Dil}(\mathscr{X} \cup\{G\}) .
$$

Для любого $s$ в качестве класса $\mathscr{X}$ рассмотрим множество полных двудольных графов с не более чем $s$ вершинами в одной из долей. Легко проверить, что

$$
\operatorname{Forb}(\mathscr{X})=\left\{C_{3}, K_{2} \oplus K_{1}, K_{s+1, s+1}\right\}
$$

и что класс $\mathscr{X}$ содержит антицепь $\left\{K_{1,2 s+1}, K_{2,2 s}, \ldots, K_{s, s+2}, \bar{K}_{2 s+2}\right\}$ длины $s+1$. Легко построить покрытие класса $\mathscr{X}$, состоящее из $s+1$ цепей. Поэтому

$$
\operatorname{Dil}(\mathscr{X})=s+1 .
$$

Вместе с тем, для любого $G \in\left\{C_{3}, K_{2} \oplus K_{1}, K_{s+1, s+1}\right\}$ совокупность графов $\left\{K_{1,2 s+1}\right.$, $\left.K_{2,2 s}, \ldots, K_{s, s+2}, \bar{K}_{2 s+2}, G\right\}$ также является антицепью. Таким образом,

$$
\operatorname{Dil}(\mathscr{X} \cup\{G\}) \geqslant s+2 .
$$

С другой стороны, класс $\mathscr{X} \cup\{G\}$ может быть покрыт системой из $s+2$ цепей. Поэтому

$$
\operatorname{Dil}(\mathscr{X} \cup\{G\})=s+2=\operatorname{Dil}(\mathscr{X})+1 .
$$

\section{3. Полные описания граничных и минимальных сложных классов для ряда задач на графах}

В [1] рассматривался вопрос о существовании минимальных сложных классов для ряда задач на графах. Там было показано, что для задачи распознавания принадлежности любому наследственному классу $\mathscr{X}$ (то есть задачи, в которой по заданному графу $G$ необходимо определить, принадлежит ли $G$ классу $\mathscr{X}$ ) минимальных сложных классов нет. Этот результат можно рассматривать как систематический способ генерации задач на графах П без минимальных сложных классов. Для этого достаточно рассмотреть наследственный класс вершинно (или реберно) 3-раскрашиваемых графов и объединить его с любым конечным наследственным классом, содержащим граф $K_{5}$. Задача распознавания принадлежности любому такому классу является $N P$-полной (поскольку задачи о вершинной и о реберной 3-раскраске имеют такой же сложностной статус). Таким образом, результат из [1] действует сразу для континуального множества задач на графах.

Возникает вопрос, нельзя ли, как в [1], получить достаточное условие существования минимальных сложных классов, также претендующее на определенную систематичность? 
В предыдущем разделе рассматривалась совокупность наследственных классов, имеющих конечное число Дилворта. Особый интерес к данным классам вызван тем обстоятельством, что любой такой сложный класс обязательно содержит некоторый минимальный сложный (и именно поэтому в предыдущем разделе все усилия были направлены на получение описания классов графов с конечным числом Дилворта). Это утверждение будет доказано далее.

Теорема 1. Если $\mathscr{X}$ является П-сложным классом и $\operatorname{Dil}(\mathscr{X})<\infty$, то $\mathscr{X}$ содержит некоторый минимальный П-сложный класс.

Доказательство. Предположим противное. Тогда существует такое бесконечное множество графов $\mathscr{Y}=\left\{G_{1}, G_{2}, \ldots\right\}$, что $G_{1} \in \mathscr{X}, G_{2} \in \mathscr{X} \cap \operatorname{Free}\left(\left\{G_{1}\right\}\right), G_{3} \in \mathscr{X} \cap \operatorname{Free}\left(\left\{G_{1}, G_{2}\right\}\right)$, $\ldots, G_{k} \in \mathscr{X} \cap \operatorname{Free}\left(\left\{G_{1}, G_{2}, \ldots, G_{k-1}\right\}\right), \ldots$, и класс $\mathscr{X} \cap \operatorname{Free}\left(\left\{G_{1}, G_{2}, \ldots, G_{k}\right\}\right)$ является П-сложным при любом $k$. Покажем, что множество 9у не может содержать бесконечных цепей. Действительно, если такая цепь существует, то все ее элементы различны и они являются порожденными подграфами некоторого графа $G$. Но это невозможно, так как $G$ содержит только конечное множество порожденных подграфов.

Заметим, что व может быть покрыто конечным множеством цепей, поскольку $\operatorname{Dil}(\mathscr{X})<\infty$. Множество У покрывается конечным множеством конечных цепей. Следовательно, это множество является конечным. Получаем противоречие. Значит, исходное предположение неверно.

В [1] были впервые предъявлены примеры минимальных сложных классов. Исследования в этом направлении были продолжены в [3, 4]. Однако во всех статьях этого цикла рассматривались задачи о списковом ранжировании (вершинный и реберный варианты) и устанавливалась минимальность некоторых сложных классов для этих задач. Вместе с тем, до настоящей работы не было известно никаких других примеров задач на графах с существованием минимальных сложных классов. Далее мы укажем новые такие случаи. Именно, будет показано, что для любого натурального $k$ существует задача на графах $\Pi_{k}$ и минимальный П ${ }_{k}$-сложный класс $\mathscr{X}_{k}$, имеющий число Дилворта, равное $k$.

Члены последовательности $\left\{\Pi_{k}\right\}-$ некоторые специальные представители задачи о наибольшем взвешенном 3-раскрашиваемом подграфе. Для любого натурального $k$ в задаче $\Pi_{k}$ заданы граф $G$ и отображение $c: E(G) \rightarrow\{-1,1\}$. Требуется найти такой подграф $H$ графа $G$, что $H$ имеет 3-раскраску вершин и величина $w(H)$ является максимально возможной. Значение $w(H)$ определяется следующим образом: $w(H)=0$, если размер наибольшего независимого множества графа $H$ не равен $k$, и

$$
w(H)=\sum_{e \in E(H)} c(e)
$$

в противном случае.

Уточним, что под сложным для задачи $\Pi_{k}$ понимается наследственный класс графов, для которого данная задача полиномиально разрешима при любой функции $c$. Ясно, что каждый $\Pi_{k}$-сложный класс содержит хотя бы один граф с наибольшим независимым множеством мощности $k$. Понятно также, что если $\mathscr{X}$ является $\Pi_{k}$-сложным, $G \in \mathscr{X}$ и $G$ имеет независимое множество мощности, превосходящей $k$, то $\mathscr{Q} \cap \operatorname{Free}(\{G\})$ также является $\Pi_{k}$-сложным. Поэтому все минимальные $\Pi_{k}$-сложные классы состоят из графов, у которых каждое независимое множество состоит из не более чем $k$ вершин. Понятно, что каждый минимальный $\Pi_{k}$-сложный класс должен быть бесконечным, поэтому любой такой класс должен содержать все клики. Это легко следует из теоремы Рамсея. 
Рассмотрим множество графов $\bigcup_{i=1}^{\infty}\left\{K_{i} \oplus \bar{K}_{s}: s \in\{0,1,2, \ldots, k-1\}\right\}$ в качестве заявленного класса $\mathscr{X}_{k}$; при этом предполагается, что $K_{i} \oplus K_{0}=K_{i}$. Очевидно, что $\mathscr{X}_{k}-$ наследственный класс графов и $\operatorname{Dil}\left(\mathscr{X}_{k}\right)=k$. Легко проверить, что $\mathscr{X}_{k}$ удовлетворяет также всем необходимым требованиям к минимальным $\Pi_{k}$-сложным классам, упомянутым в предыдущем абзаце.

Теорема 2. Для любого s класс $\mathscr{X}_{s}$ является минимальным $\Pi_{s}$-сложным.

Доказательство. Докажем сначала, что класс $\mathscr{X}_{s}$ является $\Pi_{s}$-сложным. Обозначим через У множество всех вершинно 3-раскрашиваемых графов. Очевидно, что класс У замкнут не только относительно удаления вершин, но и относительно удаления ребер. Покажем, что при любом $s N P$-полная задача распознавания принадлежности графа классу у полиномиально сводится к задаче $\Pi_{s}$ в классе $\mathscr{X}_{s}$. Отсюда следует $N P$-полнота задачи $\Pi_{s}$ в классе $\mathscr{X}_{s}$.

Рассмотрим произвольный граф $G$. Добавим к $G$ несколько ребер до полного графа и $s-1$ изолированную вершину и получим граф $G^{\prime}$. Для каждого $e \in E\left(G^{\prime}\right) \cap E(G)$ положим $c(e)=1$; для всех остальных ребер $e$ графа $G^{\prime}$ положим $c(e)=-1$. Понятно, что граф $H$ - решение задачи $\Pi_{s}$ для графа $G^{\prime}-$ не содержит ни одного ребра с отрицательным значением функции $c$, поскольку класс у замкнут относительно удаления ребер. Ясно, что граф $G$ является вершинно 3-раскрашиваемым тогда и только тогда, когда

$$
w(H)=|E(G)|
$$

Отсюда следует заявленная выше сводимость.

Докажем теперь минимальность класса $\mathscr{X}_{s}$. Для этого покажем, что для любого $G \in \mathscr{X}_{s}$ класс $\mathscr{X}_{s} \cap \operatorname{Free}(\{G\})$ является $\Pi_{s}$-простым. Среди графов из $\mathscr{L}_{s} \cap \operatorname{Free}(\{G\})$ необходимо рассмотреть только графы вида $K_{n} \oplus \bar{K}_{s-1}$, так как во всех остальных случаях в качестве ответа можно взять результат удаления всех ребер из входного графа. Пусть

$$
G=K_{i} \oplus \bar{K}_{j}
$$

Заметим, что тогда $n \leqslant i$. Следовательно, во множестве $\mathscr{X}_{s} \cap \operatorname{Free}(\{G\})$ графов вида $K_{n} \oplus \bar{K}_{s-1}$ имеется только конечное число. Поэтому задача $\Pi_{s}$ в классе $\mathscr{X}_{s} \cap \operatorname{Free}(\{G\})$ полиномиально разрешима при любом $G$.

Заметим, что класс $\mathscr{X}_{k}$ является минимальным сложным не только для задачи $\Pi_{k}$. Для образования новых таких задач достаточно перейти от рассмотрения вершинно 3-раскрашиваемых графов к графам из произвольного класса, замкнутого относительно удалений вершин и ребер, с $N P$-полной задачей распознавания принадлежности. Примерами таких классов графов являются классы вершинно $k$-раскрашиваемых и реберно $k$-раскрашиваемых графов при $k>3$.

Можно также заметить, что класс $\mathscr{X}_{s}$ не является единственным минимальным $\Pi_{s}$-сложным классом с числом Дилворта, равным $s$. Для этого через $y_{s}, s \geq 1$, обозначим совокупность графов, являющихся порожденными в графах из $\bigcup_{i=1}^{\infty}\left\{\overline{K_{s} \oplus \bar{K}_{i}}\right\}$. Легко проверить, что

$$
\operatorname{Dil}\left(\mathscr{Y}_{s}\right)=s
$$

По аналогии с доказательством теоремы 2, можно показать, что $\mathscr{Y}_{s}$ есть минимальный $\Pi_{s}$-сложный класс. 
Итак, классы $\mathscr{X}_{k}$ и $\mathscr{Y}_{k}$ являются минимальными $\Pi_{k}$-сложными. Возникает вопрос о существовании других таких классов. Представляет также интерес вопрос, каким образом устроено все множество минимальных $\Pi_{k}$-сложных классов. Для ответа на этот вопрос вначале напомним понятие расщепляемого графа. Граф $G$ называется расщепляемым, если множество его вершин $V$ можно так разбить на два подмножества $V_{1}$ и $V_{2}$, что $V_{1}$ порождает в графе полный подграф, а $V_{2}$ порождает пустой подграф. Рассмотрим всевозможные бесконечные классы расщепляемых графов, у которых размер наибольшего независимого множества равен $k$. Через $\chi(k)$ обозначим семейство наследственных замыканий таких классов. Через $\mathcal{X}^{*}(k)$ обозначим множество минимальных по включению классов из $\mathcal{X}(k)$. По аналогии с доказательством теоремы 2, можно показать, что каждый класс из $\mathcal{X}(k)$ является инимальным $\Pi_{k}$-сложным. Оказывается, что других таких классов не существует.

Теорема 3. Все множество минимальных $\Pi_{k}$-сложных классов совпадает с множеством $\chi^{*}(k)$.

Доказательство. Предположим противное. Тогда существует минимальный П ${ }_{k}$-сложный класс $\mathscr{X}$, не принадлежащий множеству $X^{*}(k)$. Рассмотрим те графы из $\mathscr{X}$, число независимости которых равно $k$. В данных графах рассмотрим подграфы, порожденные объединением наибольшего независимого множества и наибольшей клики. Понятно, что таких попарно неизоморфных расщепляемых подграфов бесконечное количество. Обозначим это множество У. Ясно, что наследственное замыкание класса У включает некоторый элемент $\mathscr{L} \in X^{*}(k)$. Но тогда $\mathscr{L} \subset \mathscr{X}$, и поэтому $\mathscr{L}$ не является минимальным сложным. Получаем противоречие. Поэтому исходное предположение неверно.

Отметим, что теорема 3 является первым примером полного описания минимальных сложных классов. Ранее результатов такого рода не удавалось получить. Возникает вопрос, как быть с $\Pi_{k}$-граничными классами. Оказывается, что все множество граничных классов для задачи $\Pi_{k}$ совпадает с множеством $\mathcal{X}^{*}(k)$. Доказательство этого утверждения составляет оставшуюся часть.

Сначала введем понятия характеристических векторов графа и класса. Для любого расщепляемого графа $G$, имеющего наибольшее независимое множество размера $k$, существуют такие непересекающиеся множества вершин $V_{1}$ и $V_{2}$, что $V(G)=V_{1} \cup V_{2}, V_{1}$ порождает в $G$ пустой подграф и содержит ровно $k$ вершин, а $V_{2}$ порождает полный подграф. Занумеруем некоторым образом все подмножества множества $V_{1}$ числами от 1 до $2^{k}$. Характеристическим вектором графа $G$ назовем такой вектор $\left(v_{1}(G), v_{2}(G), \ldots, v_{2}(G)\right)$, в котором $v_{i}(G)$ - количество тех вершин $x \in V_{2}$, которые имеют окрестность $V_{2} \backslash\{x\} \cup V^{(i)}$, где $V^{(i)}$ есть $i$-е подмножество множества $V_{1}$. Легко проверить, что характеристический вектор - инвариант графа $G$, поскольку он не зависит от выбора пары $\left(V_{1}, V_{2}\right)$. Понятно также, что любой граф с наибольшим независимым множеством размера $k$ имеет единственный характеристический вектор, и, наоборот, любой вектор из $\mathbf{Z}_{+}^{2^{k}}$ является характеристическим для некоторого такого графа.

Пусть $\mathscr{X}$ - наследственное замыкание некоторого множества графов 9, каждый из которых имеет наибольшее независимое множество мощности $k$. Характеристическим вектором класса $\mathscr{X}$ называется вектор $\left(v_{1}(\mathscr{X}), v_{2}(\mathscr{X}), \ldots, v_{2^{k}}(\mathscr{X})\right)$, где под $v_{i}(\mathscr{X})$ понимается бесконечность, если величина $v_{i}(G)$ может принимать бесконечно большие значения, или число $\max _{G \in \mathscr{X}} v_{i}(G)$ в противном случае. Из наследственности класса $\mathscr{X}$ следует, что $\mathscr{y}$ состоит из тех и только тех графов $H$, которые имеют такой характеристический вектор $\left(v_{1}(H), v_{2}(H), \ldots, v_{2^{k}}(H)\right)$, что для любого $i \in\left\{1,2, \ldots, 2^{k}\right\}$ выполнено неравенство

$$
v_{i}(H) \leqslant v_{i}(\mathscr{X})
$$


бесконечность, как обычно, считается больше любого числа.

Понятие характеристического вектора класса и теорема Диксона [8] позволяют доказать важное вспомогательное утверждение - конечность множества $X^{*}(k)$. Теорема Диксона состоит в том, что при любом фиксированном $n$ на множестве $\mathbf{Z}_{+}^{n}$ отношение порядка “быть покоординатно не больше" имеет лишь конечные антицепи. Аналог теоремы Диксона может быть легко доказан и для можества $\left(\mathbf{Z}_{+} \cup\{\infty\}\right)^{n}$.

Лемма 4. При любом $k$ множество $\chi^{*}(k)$ конечно.

Доказательство. Рассмотрим характеристические вектора классов из $\mathcal{X}^{*}(k)$. Поскольку любые два таких класса не сравнимы по отношению включения, на множестве $\left(\mathbf{Z}_{+} \cup\{\infty\}\right)^{n}$ множество всех рассматриваемых характеристических векторов образует антицепь по отношению “быть покоординатно не больше”. Из аналога теоремы Диксона следует конечность этой антицепи.

Теорема 4. Для любого $k$ все множество $\Pi_{k}$-граничных классов совпадает с множеством $X^{*}(k)$.

Доказательство. Поскольку каждый класс $\mathcal{X}^{*}(k)$ является $\Pi_{k}$-сложным, то он также является и $\Pi_{k}$-предельным. Докажем, что других предельных классов нет. Предположим противное; тогда существует такой $\Pi_{k}$-предельный класс $\mathscr{X}$, что $\mathscr{X} \notin \mathcal{X}^{*}(k)$. При доказательстве теоремы 3 фактически было доказано, что любой $\Pi_{k}$-сложный класс содержит некоторый класс из $X^{*}(k)$. Отсюда следует, что каждый элемент любой монотонно убывающей последовательности из $\Pi_{k}$-сложных классов включает хотя бы один класс из $X^{*}(k)$. Так как совокупность $X^{*}(k)$ является конечной, то существует подпоследовательность, каждый член которой включает один и тот же класс из $X^{*}(k)$. Поэтому и предел любой монотонно убывающей последовательности из $\Pi_{k}$-сложных классов включает хотя бы один класс из $\chi^{*}(k)$. Отсюда следует, что все множество $\Pi_{k}$-предельных классов совпадает с множеством $X^{*}(k)$. Отсюда также следует минимальность классов из $X^{*}(k)$, то есть, что каждый класс из $\mathcal{X}^{*}(k)$ действительно является $\Pi_{k}$-граничным.

Задачи из множества $\left\{\Pi_{1}, \Pi_{2}, \ldots\right\}$ - первые примеры задач на графах с полным описанием граничных классов. Это также и первые примеры задач, для которых доказана конечность множества граничных классов.

\section{Список литературы}

1. Малышев Д. С., О минимальных сложных классах графов. Дискретный анализ и исследование операчий (2009) 16, №6, 43-51.

2. Малышев Д. С., Исследование грании эффективной разрешимости в семействе наследственных классов графов, Дисс. на соискание уч. степени канд. физ.-мат. наук, 01.01.09- Дискретная математика и математическая кибернетика, Нижний Новгород, 2009.

3. Малышев Д. С., Последовательные минимумы решетки наследственных классов графов для задачи о реберном списковом ранжировании. Вестник Нижегородского унив. (2010) №4, 143146.

4. Малышев Д. С., Минимальные сложные классы графов для задачи о реберном списковом ранжировании. Дискретный анализ и исследование операциий (2011) 18, №1, 70-76.

5. Alekseev V. E., On easy and hard hereditary classes of graphs with respect to the independent set problem. Discrete Appl. Math. (2004) 132, 17-26. 
6. Alekseev V. E., Boliac R., Korobitsyn D. V., Lozin V. V., $N P$-hard graph problems and boundary classes of graphs. Theoret. Comput. Sci. (2007) 389, 219-236.

7. Alekseev V. E., Korobitsyn D. V., Lozin V. V., Boundary classes of graphs for the dominating set problem. Discrete Mathematics (2004) 285, 1-6.

8. Dickson L. E., Finiteness of the odd perfect and primitive abundant numbers with $n$ distinct prime factors. Amer. J. Math. (1913) 35, 413-422.

9. Kaminski M., New algorithmic and hardness results on graph partitioning problems, PhD Thesis. Rutgers Center for Operations Research, Rutgers Univ., New Jersey, 2007.

10. Lozin V. V., Boundary classes of planar graphs. Combinatorics, Probability, and Computing (2008) 17, 287-295.

11. Millanic M., Algorithmic developments and complexity results for finding maximum and exact independent sets in graphs, PhD Thesis. Rutgers Center for Operations Research, Rutgers Univ., New Jersey, 2007.

12. Perles M., On Dilworth's theorem in the infinite case. Israel J. Math. (1963) 1, 108-109.

Статья поступила 7.07.2011. 